\title{
« Les Mots de l'histoire » et la Begriffsgeschichte / sémantique historique
}

\section{Michael Werner}

\section{OpenEdition}

\section{Journals}

Édition électronique

URL : http://journals.openedition.org/ifha/460

DOI : 10.4000/ifha.460

ISSN : 2198-8943

\section{Éditeur}

IFRA - Institut franco-allemand (sciences historiques et sociales)

\section{Édition imprimée}

Date de publication : 30 septembre 2012

Pagination : 187-194

ISSN : 2190-0078

\section{Référence électronique}

Michael Werner, « «Les Mots de l'histoire » et la Begriffsgeschichte / sémantique historique », Revue de I'IFHA [En ligne], 4 | 2012, mis en ligne le 14 février 2013, consulté le 20 avril 2019. URL : http:// journals.openedition.org/ifha/460 ; DOI : 10.4000/ifha.460

Ce document a été généré automatiquement le 20 avril 2019

(CIFHA 


\title{
«Les Mots de l'histoire » et la Begriffsgeschichte / sémantique historique
}

\author{
Michael Werner
}

Renvoyer à la Begriffsgeschichte et à la sémantique historique pour circonscrire la genèse des Mots de l'histoire semble une évidence. Ces deux courants historiographiques ne thématisent-ils pas les rapports variés des mots et des choses qui étaient au cœur du projet intellectuel ? Or, à y regarder de plus près, l'affaire était à la fois plus complexe et plus longue à se décanter. D'abord parce que le projet est parti, à l'origine, d'un séminaire d'histoire et d'historiographie allemandes organisé à partir de 2001 par des historiens des Universités de Paris-I, Paris-VII et de l'EHESS travaillant sur l'Allemagne ou avec des collègues allemands. Par sa configuration et par le regard porté sur l'objet, ce séminaire était, de fait, une entreprise d'historiographie franco-allemande comparée. Se proposant de confronter les historiographies, il était peu attentif aux «mots». L'intérêt pour les différences entre les concepts mis en œuvre par les historiens ne s'est imposé que progressivement, ce qui a conduit les organisateurs, dont le cercle s'était élargi, à concevoir un premier retour réflexif sur les outils conceptuels et proposer la formule des «Mots de l'histoire: historiens français et allemands face à leurs sources et à leurs outils». À partir de ce moment, l'objectif consistait, pour le dire vite, de combiner historiographie et sémantique historique. Parallèlement, l'approche comparative était complétée par la prise en considération des interactions, traductions, transferts et transpositions, induisant un dispositif analytique d'histoire croisée.

Le deuxième retour réflexif était propre au sujet de la Begriffsgeschichte/sémantique historique. Car en tant que courant historiographique décliné de façon différente dans les deux pays, la sémantique historique s'est imposée elle-même comme thème des « Mots de l'histoire ». Le 6 janvier 2006, une séance de séminaire animée par Otto Gerhard Oexle, Peter Schöttler, Alexandre Escudier et Joseph Morsel a été consacrée à la «Genèse comparée de la sémantique historique ». Tout en étant davantage centrée sur la lexicométrie politique, la question de l'analyse des discours et les rapports entre 
Begriffsgeschichte et histoire-problème, elle avait la particularité d'interroger les différents courants de sémantique historique à travers des questionnements analytiques propres à la sémantique historique. Celle-ci était à la fois objet et méthode. Ce dédoublement ne constitue, certes, qu'une radicalisation de toute démarche historiographique qui consiste à faire l'histoire de l'histoire. Mais il comporte ici, sur le plan épistémologique, une trappe d'auto-référentialité, une forme de mise en abîme. Car on pourrait aujourd'hui faire l'expérience d'une sorte de séance au second degré visant, à propos du couple " Begriffsgeschichte/sémantique historique " et avec les outils proposés par les différents courants d'histoire conceptuelle de Koselleck, l'historicisation de l'historicisation de l'histoire des concepts dans le paysage franco-allemand!

Nous optons dans ces quelques remarques pour une approche plus terre à terre, en tentant de préciser les relations des "Mots de l'histoire" avec deux pôles de la sémantique historique: la Begriffsgeschichte de Koselleck et le programme d'histoire comparée des terminologies historiennes dessiné par Marc Bloch. Commençons par le second.

Dans sa célèbre conférence devant l'association internationale des historiens en 1928, Marc Bloch avait appelé à une histoire comparée de l'Europe. Ce programme ne visait pas seulement l'histoire sociale, mais comportait également, on l'a souvent oublié, un travail comparatif sur les concepts de l'historien. Partant du décalage entre les termes de " Höriger » et "tenancier», Bloch a indiqué la difficulté des communautés historiennes nationales à s'entendre à travers des terminologies disparates. Il a, en outre, signalé - et cela a été important dans la gestation du projet des "Mots de l'histoire»-que le problème se situait en réalité à deux niveaux: celui des sources et celui des historiographies. Et c'est, selon lui, aux dernières qu'incombe la responsabilité principale des difficultés évoquées: "Nous avons, à tort ou à raison, et plus ou moins inconsciemment, élaboré des vocabulaires techniques. Chaque école nationale a construit le sien, sans se préoccuper du voisin. L'histoire européenne est ainsi devenue une véritable Babel. » Et de signaler également les différences entre les questionnaires historiographiques élaborés par les écoles nationales. D'où son appel pour une "réconciliation de nos terminologies et de nos questionnaires »' Cette réconciliation devait nécessairement passer par des enquêtes historiographiques comparatives à l'instar de celle qui a été lancée par Lucien Febvre et Henri Berr, lors de la première Semaine de synthèse de mai 1929, à propos de la notion de civilisation ${ }^{2}$. Mais force est de constater qu'en France, ces initiatives sont restées isolées et n'ont pas connu de véritables suites. Le programme de Marc Bloch restait donc d'actualité, surtout si on le rapportait à la question du nationalisme méthodologique dénoncé par le sociologue Ulrich Beck. D'un côté il y avait donc cette question du rapport entre la langue des sources et la «terminologie » de l'historien, pour reprendre l'expression de Marc Bloch. Elle posait le problème de la langue, de son historicité et de la perméabilité entre les différents souschamps qui la composent (ici: langue courante, langue des acteurs, langue des spécialistes qui tentent de stabiliser leurs mots). De l'autre, la nécessité de prendre en compte la pluralité des langues nationales et l'incidence de cette pluralité sur l'évolution des historiographies respectives. Par ailleurs, la perspective de la "réconciliation » des terminologies était à compléter, pour la programmation des "Mots », par une analyse non seulement des différences, mais aussi des interactions et des interdépendances.

Pour l'autre source d'inspiration et de confrontation, la tradition de la Begriffsgeschichte, à laquelle d'aucuns, parmi les organisateurs des « Mots de l'histoire », s'étaient confrontés 
depuis le début des années $1990^{3}$, les interférences étaient évidentes. Les apports qu'elle a pu fournir à la conception et la mise en œuvre de l'initiative sont évidents, à la fois sur le plan théorique et pour la pratique de l'enquête.

La Begriffsgeschichte développée par Koselleck repose sur une hypothèse fondamentale concernant les relations entre la langue et le social: les deux sont des champs en transformation permanente, à l'évolution fortement corrélée. Par rapport au monde social en général, la langue a l'avantage de pouvoir être appréhendée pour ainsi dire directement. Elle fournit un matériau de première main, que l'on peut observer en tant que tel. Son évolution est un précieux indicateur des transformations du social. La création de nouveaux concepts ainsi que la transformation incessante de leur signification (c'est ce que Koselleck appelle la structure temporelle de la langue) permettent de saisir le changement social. Ceci dit, la Begriffsgeschichte ne se fonde pas sur un dualisme langue versus monde social (ou mots versus choses). Elle emprunte à la linguistique le modèle du triangle sémiotique. Ses trois points d'angle sont constitués par le symbole (ou signe ou mot), le concept (ou référence) et le référent (l'objet). Koselleck a légèrement modifié le triangle en distinguant parole-concept (Wort, mais aussi Begriff), signification (Bedeutung) et réalité (soziale Wirklichkeit, Objekte), opération d'ailleurs pas vraiment correcte pour les linguistes. Pour lui, l'histoire des concepts n'est cependant pas simplement un reflet de l'histoire sociale. Elle suit une dynamique propre d'appropriation et de réinterprétation par les acteurs, notamment autour des Kampfbegriffe et des Bewegungsbegriffe qui sont au centre de l'analyse de Koselleck. Enfin, s'agissant de la question de la pluralité des langues et, subséquemment, de la traduction, de l'intraduisibilité, de la circulation etc., la Begriffsgeschichte a certes vu le problème qui en résulte. Koselleck lui-même l'a signalé et a suggéré quelques pistes, en insistant sur la "programmation sémantique » spécifique induite par l'histoire dans une langue donnée ${ }^{4}$. Mais il n'est pas allé beaucoup plus loin. Les articles des Geschichtliche Grundbegriffe sont développés, dans leur très grande majorité, à partir d'entrées allemandes et n'interrogent pas systématiquement les cheminements plurilingues et supra-nationaux des concepts (abstraction faite, le cas échéant, des origines grecques ou latines). Elle n'a donc pas vraiment pris la mesure des problèmes d'une histoire transnationale des concepts, de leurs interactions et des interdépendances qui se sont tissées au cours de l'histoire.

Cette dimension a été prise en compte par deux autres entreprises, le Vocabulaire européen des philosophies dirigé par Barbara Cassin et le Dictionnaire des concepts nomades en sciences sociales coordonné par Olivier Christin. Le projet ambitieux de B. Cassin est parti de la question des intraduisibles et accentue, de ce fait, les différences dans l'évolution des concepts spécifiques à une culture et une langue. Le dictionnaire d'O. Christin, d'ailleurs postérieur à l'initiative des "Mots de l'histoire", est davantage centré sur la circulation et la réappropriation des concepts. Inspiré de la sociologie des champs de Bourdieu, il s'intéresse aux usages des concepts à travers une réflexion historique et sociologique portant sur les producteurs et les adaptateurs. Le choix des entrées est assez éclectique. Mais l'un des intérêts de l'entreprise est qu'elle refuse les deux pièges complémentaires : réduire la réalité socio-historique à sa dimension linguistique ou discursive et considérer 
que les migrations et transpositions des concepts seraient solubles dans les contextes socio-historiques.

Mais revenons aux apports de la Begriffsgeschichte au projet des « Mots de l'histoire ». Ils peuvent se résumer ainsi :

1. L'histoire des concepts met l'accent sur l'un des problèmes centraux de toute investigation historique : la question du contexte. Cette question a été davantage théorisée par l'École de Cambridge que par Koselleck lui-même. La reconstitution des contextes d'élaboration, d'usage et de transmission fournit la trame première d'une histoire des mots de l'histoire. Koselleck considère la question des contextes d'un point de vue pragmatique. Les contextes sont théoriquement illimités. C'est au chercheur de sélectionner ceux qui, pour la question posée, lui semblent pertinents. Plus il élargit le focus, plus il enrichit les significations à prendre en considération, tout en risquant de diluer les noyaux sémantiques et les lignes de différenciation.

2. Le volet complémentaire de la question du contexte est celui du changement de signification. Pour Koselleck, les concepts (Begriffe) contiennent toujours plus que les significations que les locuteurs leur attribuent dans une situation donnée. C'est ce qu'il appelle le Bedeutungsüberschuss, le surplus de significations des concepts. Les concepts transportent une ou plusieurs histoires, et ils changent de sens.

3. L'historien se trouve face à une pluralité de concepts et à des systèmes de concepts. Ceci a pour conséquence qu'il est vain d'analyser un concept isolé, sans tenir compte des concepts voisins (les champs sémantiques) et des concepts opposés (les Gegenbegriffe de Koselleck). Cette thèse fondatrice a été très importante pour le programme des «Mots", à la fois par rapport au contexte spécifique des langues et traditions nationales, et au regard des interactions transnationales. Dans les deux cas, il fallait tenter de démêler l'histoire croisée des processus de co-constitution des concepts et des catégories. Cette dernière dimension, transnationale, était, nous l'avons dit, peu présente chez Koselleck, mais elle est aujourd'hui prise en compte par différents réseaux de recherche internationaux et des revues comme Contributions to the History of Concepts.

4. La distinction, capitale entre Wort (parole) et Begriff (concept). Selon Koselleck, la parole est toujours singulière (en tant qu'acte d'énonciation ou choix d'écriture), alors que le Begriff inscrit la parole singulière dans un système de représentations, une chaine de significations historiquement construite. Il en résulte ce que Koselleck appelle la structure temporelle des Begriffe. Celle-ci fait des concepts des objets privilégiés de l'historien. Appliquée au domaine de l'historiographie, elle ouvre des pistes d'investigation particulièrement prometteuses.

Inspiré par le courant de la sémantique historique, le projet des « Mots de l'histoire » s'en écarte cependant sur un certain nombre de points. J'en ferai ressortir quatre qui me paraissent devoir être retenus :

1. La « réalité » à laquelle les coordinateurs de l'entreprise ont essayé de raccorder l'évolution des concepts n'était pas, au premier chef, le social, le politique ou le culturel, mais bien davantage les mondes académiques avec leurs enjeux propres, à la fois intellectuels et institutionnels. Il n'était donc pas besoin de dresser des légitimations particulières face aux exigences de l'histoire sociale et culturelle - il suffisait d'insister sur les apports réflexifs de l'expérience et ses effets sur les pratiques des historiens impliqués. Questionner les présupposés historiographiques respectifs, mettre en question ce qui semble aller de soi dans une tradition marquée par le cadrage national pouvait constituer une justification à elle seule.

2. La limitation au noyau franco-allemand était une restriction utile, féconde, même si, au fil des séances, elle a souvent été dépassée par des excursions dans les domaines anglo-saxon et italien, notamment. Comme pour d'autres pans de l'histoire intellectuelle européenne, le franco-allemand constitue une sorte de prisme permettant de faire ressortir des lignes de 
force et d'analyser des spectres plus larges. Ce « saut de côté » franco-allemand, qui produit un effet de décentrage important, est - comme l'expérience le montre - particulièrement riche en effets de sens.

3. La problématisation de la frontière entre langage savant et langue courante a dû être poussée bien plus loin que ce n'était le cas pour la Begriffsgeschichte classique. Koselleck a bien vu la question, puisqu'il distingue, en principe, Wort et Begriff, mais il reste, sur le fond, attaché à l'étude des cimes représentées par les grands concepts fondamentaux. Pour l'historien, bien plus que pour le sociologue et l'anthropologue qui disposent de terminologies davantage stabilisées, le problème de cette frontière est capital. Il y est confronté tant dans son travail quotidien que sur le plan épistémologique. Pour lui, cette frontière est bien poreuse. Les acteurs s'emparent des notions et concepts des historiens, et, inversement, les concepts des historiens sont constamment travaillés, nourris et déformés par la pratique des acteurs ainsi que par l'évolution sémantique propre à la vie des langues. Nous en avons vu des exemples nombreux au cours des séminaires, des couples «Landschaft / Paysage » à « Hören / Ecoute », en passant par « Wallfahrt / Pèlerinage ».

4. Ceci fait retour, malgré tout (et en particulier malgré le point 1) sur la frontière entre l'histoire comme discipline et forme organisée de savoir et le «réel », qu'il soit politique, social, culturel ou académique - ou tout cela à la fois. Dans le domaine de l'historiographie croisée des concepts, les répartitions ne se font pas de façon habituelle, attachée à maintenir la distinction entre res facta et res gesta. L'analyse des interactions entre les deux sphères s'oriente davantage vers une analyse de l'ancrage social, politique et culturel des historiographies qui a été l'un des fils rouges poursuivis à travers les séances.

Qu'il me soit permis de revenir brièvement, en conclusion, sur la question, posée au début, d'une sémantique historique des historiographies, exposée à la boucle infinie de l'auto-référentialité. Elle pose de manière exemplaire le problème des paradoxes de l'historicisation. D'un côté celle-ci est, sur le plan épistémologique, un processus illimité, sans fin possible. En l'occurrence, il s'agissait, dans l'exemple des rapports entre les "Mots de l'histoire » et la Begriffsgeschichte, mais aussi, au fond, dans toute l'entreprise des « Mots ", d'historiciser le travail de l'historien en historicisant ses outils analytiques et discursifs (les Begriffe). Mais en même temps, les intérêts de connaissance ( Erkenntnisinteressen) des uns et des autres, organisateurs, intervenants et participants, posent, heureusement, des limites à la fois pragmatiques et scientifiques (ou scientifiquement défendables) au déroulement du processus d'historicisation. L'objectif de l'entreprise était de trouver un niveau d'articulation praticable et, en même temps, le plus éclairant possible entre les deux logiques d'historicisation et de fertilisation pour les besoins du présent. En ce sens, me semble-t-il, les « Mots de l'histoire » ont pu jouer le rôle d'un laboratoire de réflexivité.

\section{NOTES}

1. Marc Bloch, "Pour une histoire comparée des sociétés européennes ", in Revue de synthèse historique, 46 (1928), p. 15-50, ici p. 48 et suivantes.

2. Centre international de synthèse, Première semaine internationale de synthèse, deuxième fascicule. Civilisation, Le mot et l'idée, Paris : La Renaissance du Livre, 1930. 
3. Voir, entre autres, le volume Reinhart Koselleck, L'expérience de l'histoire, ouvrage édité et préfacé par M. Werner, Paris : Gallimard, Le Seuil, 1997, dont l'essentiel des traductions a été assuré par Alexandre Escudier.

4. Reinhart Koselleck, "Hinweise auf die temporalen Strukturen begriffsgeschichtlichen Wandels ", in : Hans-Erich Bödeker (dir.), Begriffsgeschichte, Diskursgeschichte, Metapherngeschichte, Göttingen : Wallstein-Verlag, 2002, p. 29-47, en particulier p. 40-42.

\section{AUTEUR}

\section{MICHAEL WERNER}

Michael Werner est directeur du Centre de Recherches interdisciplinaires sur l'Allemagne (CRIA, UMR CNRS/EHESS 8131) à l'École des Hautes Études en Sciences Sociales. 